\title{
FEED DRIVE CONDITION MONITORING USING MODAL PARAMETERS
}

\author{
J. Ellinger ${ }^{1 *}$, T. Semm ${ }^{1}$, M. Benker ${ }^{1}$, P. Kapfinger ${ }^{1}$, R. Kleinwort ${ }^{1}$, M. F. Zäh ${ }^{1}$ \\ ${ }^{1}$ Technical University Munich, Institute for Machine Tools and Industrial Management, Munich, Germany \\ *Corresponding author; e-mail: Johannes.Ellinger@iwb.mw.tum.de
}

\begin{abstract}
Ball screws and linear guides are among the key components of machine tools. Abrasive wear causes a loss in stiffness of these components over time affecting the attainable manufacturing precision and, eventually, leads to failures and costly down-time. In order to control these effects, the condition of the crucial feed drive components needs to be monitored. This paper shows, how the feed drive condition can be monitored by looking at the modal parameters of the system. It will be shown, that preload loss cannot only be detected globally, but can be traced back to the worn component. A distinct test cycle was developed for this purpose.
\end{abstract}

\section{Keywords:}

Feed drive; Condition monitoring; Ball screw; Linear guide; Preload loss; Analysis of variance; Machine tool

\section{INTRODUCTION}

The productivity of machine tools is mainly characterized by the machine's chip removal rate [Zaeh et al. 2017] and the availability of the machine itself [Verl et al. 2009]. For both factors, the feed drive components ball screw and linear guides are crucial. On the one hand, they are the critical components regarding failure [Walther 2011] and on the other hand their stiffness strongly affects the position accuracy and thus the manufacturing precision [Maier 2015]. The decisive factor here is the preload of these components, which is needed to increase stiffness and prevent backlash. The degradation of ball screws and linear guides due to abrasive wear of their roller elements leads to a successive loss of preload. In the past, mainly reactive and time-based maintenance approaches have been used to secure productivity [Zhai \& Reinhart 2018]. In the first case, maintenance is performed after a failure has occurred. In the second case, maintenance is done according to a fixed schedule. Both approaches lead to a conflict of objectives in terms of profitability, since either costly downtime must be tolerated, or maintenance actions are taken too early and resources are wasted.

A promising new approach is condition-based maintenance ("condition monitoring"), where maintenance activities are linked to the actual wear state of components [Farrar \& Worden 2012]. This approach promises to increase machine availability while significantly reducing maintenance costs by eliminating unnecessary maintenance, using synergy effects by maintaining multiple similar machines in parallel and selecting an optimal maintenance time.

In order to be able to do so, this paper shows how to detect the preload loss of balls screws and linear guides using features, that describe the dynamic behavior of machine tools.
First, in section 2 a short overview is given regarding existing methods to detect a preload loss of feed drives. Section 3 states the importance of the modal parameters on the dynamic behavior of linear systems by summarizing basics of structural dynamics. In section 4 it is shown, how the knowledge gained from an FEM analysis can be used to not only detect preload loss, but to also locate it. In section 5 a test cycle is presented enabling reliable and reproducible dynamic measurements. Section 6 contains two methods for isolating wear sensitive features from the measured system's dynamic behavior. The sensitivity of those features is proven in section 7 with the help of a variance analysis.

\section{FEED DRIVE CONDITION MONTITORING}

In literature, a variety of different methods exist for the condition monitoring of feed drive components. In Tsai et al. [2014] it is shown, that the ratio of sliding and rolling of the roller elements in ball screws and thus the ball passing frequency is dependent on the friction caused by the preload. By placing an accelerometer near the return tube and implementing a Vold-Kalman-filtering-order-trackingalgorithm, the current preload level can be monitored. A similar approach is followed by Feng \& Pan [2012], who have shown the correlation between the ball screw preload level and the vibrational behavior of the feed drive system both in theory and practice using an analytic four degree-of-freedom model and a feed drive test bench, respectively. Abstaining from the use of external sensors, Chang et al. [2010] were able to distinguish between two different preload levels by simply measuring the feed drive motor current. To do so, a combination of empirical mode composition (EMD) and multiscale entropy (MSE) methods was used. A similar approach was implemented in Verl et al. [2009] using the vibration energy spectrum as a wear 
sensitive feature. In contrast to the presented approaches, the concept of a "characteristic distance", presented in Maier [2015] is not dependent on a distinct test cycle with constant feed rate, but can also capture ball screw preload loss. In Hillenbrand et al. [2018] strain sensors were embedded in the ball screw nut. This way, by looking at the RMS value of the strain signal, new and worn ball screws were distinguished.

In contrast to the other work in this field, here the current state of preload is determined by directly evaluating the measured dynamic behavior of the feed drive system. This way, the detection of preload loss is not only possible on a global level, but can also be done with respect to single feed drive components. Furthermore, the definition of a threshold, at which maintenance actions must be conducted, can be supported by comparing the required machining precision to the actual machining precision. This is possible since the latter is strongly governed by the machine tool's dynamic behavior.

\section{MODAL DECOMPOSITION}

The behavior of real systems excited by the force $f$ can be described as a multiple-degree-of-freedom (MDOF) model in various directions [Altintas 2012]:

$$
\left[\boldsymbol{M}_{\boldsymbol{x}}\right]\{\ddot{\boldsymbol{x}}\}+\left[\boldsymbol{C}_{\boldsymbol{x}}\right]\{\dot{\boldsymbol{x}}\}+\left[\boldsymbol{K}_{\boldsymbol{x}}\right]\{\boldsymbol{x}\}=\{\boldsymbol{f}\}
$$

The mass matrix $\left[\boldsymbol{M}_{\boldsymbol{x}}\right]$, the viscous damping matrix $\left[\boldsymbol{C}_{\boldsymbol{x}}\right]$ and the stiffness matrix $\left[\boldsymbol{K}_{x}\right]$ have the dimension $n \times n$, where $n$ is the number of degrees of freedom. The vectors $\ddot{x}, \dot{x}, x$ describe the accelerations, speeds and displacements. The equation of motion for this system is represented in the frequency domain by using the well-known Fourier transformation, leading to

$$
\left(-\left[\boldsymbol{M}_{\boldsymbol{x}}\right] \omega^{2}+\left[\boldsymbol{C}_{\boldsymbol{x}}\right] j \omega+\left[\boldsymbol{K}_{\boldsymbol{x}}\right]\right)\{X(j \omega)\}=\{F(j \omega)\}
$$

By rearranging equation (2) the following transfer function representation is obtained:

$$
[H(j \omega)]=\frac{\{X(j \omega)\}}{\{F(j \omega)\}}=\sum_{k=1}^{n} \frac{[R]_{k}}{(j \omega)^{2}+j 2 D_{k} \omega_{n, k} \omega+\omega_{n, k}^{2}}
$$

Here, $\omega_{n, k}$ and $D_{k}$ are the natural frequencies and the modal damping ratio for mode $k$ of the system and $[R]_{k}$ reflects the residues of mode $k$. These are the modal parameters for the MDOF-System.

It can be seen, that the dynamic behavior of a system can be fully described with just a few variables in the form of the modal parameters. In most cases not all modes $k$ are needed to describe the dynamic behavior properly [Semm et al. 2018]. Observing the modal parameters can thus be seen as a storage-efficient way for monitoring a system's dynamic behavior. More important, for feed drives each of the lower structural modes represents vibrations caused by one or two substructures [Zaeh et al. 2004]. Thus, the condition of these components can be identified by monitoring the related modes and their modal parameters.

\section{FEM ANALYSIS}

By using a FEM simulation model developed in Zaeh et al. [2019], the structural behavior of a feed drive system can be predicted. Even though non-linear friction is linearized by using linear stiffnesses as a replacement and the model is not perfectly parametrized for simulating the feed drive test bench presented in section 5, the model can be regarded as close to the real structure. In this work, the focus is on a proof of concept rather than on a perfect match between simulation and reality.
In the model, the ball screw and the linear guides are modeled as an assembly of multiple springs and dampers each [Zaeh et al. 2004]. It is assumed, that a loss of preload does not change the damping values and uniformly affects all spring stiffnesses. Therefore, preload loss can simply be simulated by proportionally reducing the stiffness values of all involved spring elements.

In order to be able to efficiently simulate the wear effects, a sub-structural coupling approach [Semm et al. 2018, Semm et al. 2019] was implemented in Nastran featuring the following components (see Fig. 1):

$$
\begin{array}{ll}
\text { - } & \text { cross slide } \\
\text { - } & \text { spindle slide } \\
\text { - } & \text { bed table }
\end{array}
$$

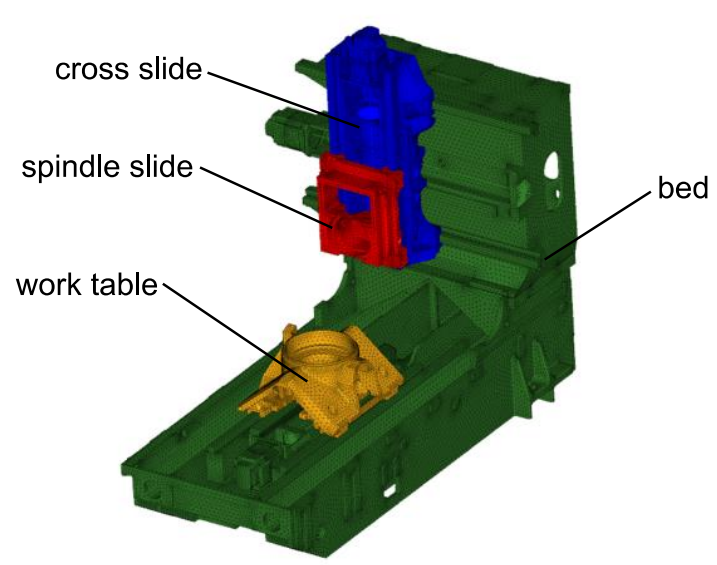

Fig. 1: Substructures for the investigated test bench (Machining Center DMG DMU55H).

The preload loss of the ball screw and the linear guide was investigated for the $\mathrm{x}$-axis of the test bench, which belongs to the cross slide component (see Fig. 7). The different preload conditions could then be analyzed by calculating a state space model of the machine tool with the sub-structural coupling of all components.

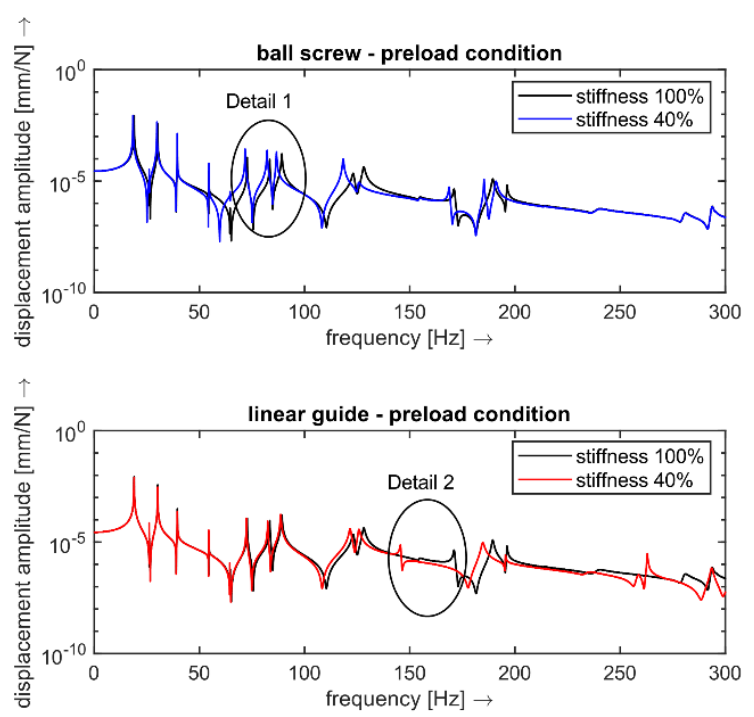

Fig. 2: Change in response to a stiffness change of all involved spring elements of the ball screw and the linear guide in the frequency range $0-300 \mathrm{~Hz}$.

Fig. 2 shows the simulated transfer function for $100 \%$ stiffness and $40 \%$ stiffness of the ball screw and the linear guide, respectively. When analyzing the machine's 
structural modes, the relevant frequency range was set to 0-300 Hz. For higher frequencies, only local structural modes were excited whose amplitudes are very small.

It can be seen, that the stiffness loss of the components impacts each mode differently. Peaks in the dynamic response tend to shift to lower natural frequencies for decreasing stiffness. The amplitude, however, can change in both directions. The goal of this analysis is to capture relevant ball screw and linear guide modes. Therefore, al modes were analyzed in more detail separately. In Fig. 2 two sections are highlighted: The range $65-100 \mathrm{~Hz}$ (Detail 1) and $130-175 \mathrm{~Hz}$ (Detail 2). In Fig. 3, the change of the natural vibration modes with the modification of the stiffness of the ball screw and the linear guide can be seen in more detail for the first frequency section.

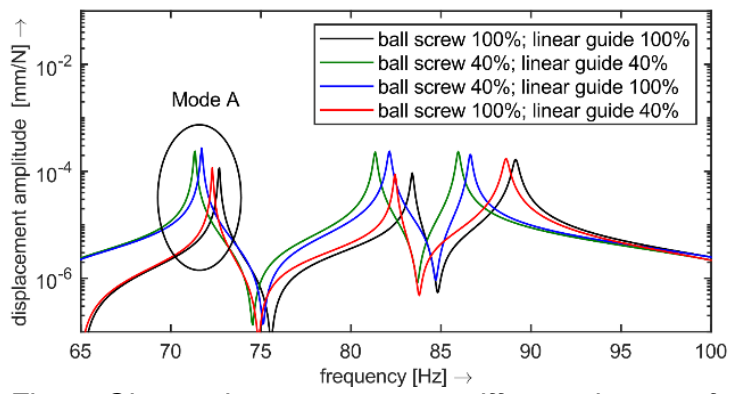

Fig. 3: Change in response to a stiffness change of all involved spring elements of the ball screw and the linear guide in the frequency range $65-100 \mathrm{~Hz}$.

It becomes clear, that stiffness loss of just the linear guide in this range has a low influence on the dynamic behavior of the machine tool since its amplitude response at the resonances follows the amplitude of the original state with full stiffness of all components. In contrast, the curve showing only ball screw stiffness loss is closer to the trajectory of the worn state meaning that the ball screw stiffness has more influence here. Fig. 4 shows the mode shape and its change in natural frequency caused by the stiffness loss of the components for the highlighted mode.
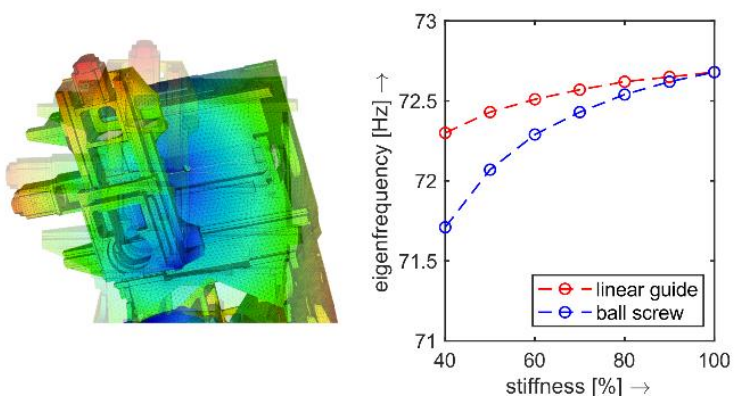

Fig. 4: Mode shape and change of natural frequency to a stiffness changes of the ball screw and the linear guide for mode $A$ (see Fig. 3).

Again, it can be seen that the influence of the linear guide in this frequency range is low compared to the influence of the ball screw since the natural frequency of the depicted mode (see Fig. 4) is more sensitive to a reduction of the ball screw stiffness than to a decrease of the linear guide stiffness.

In the frequency range of $130-175 \mathrm{~Hz}$ the stiffness of the linear guide has a more significant influence on the machine's dynamic behavior than the stiffness of the ball screw. Here, the response of reduced linear guide stiffness and $100 \%$ stiffness of the ball screw is close to the worn state. The combination of reduced ball screw stiffness and $100 \%$ stiffness of the linear guide follows the trajectory with full stiffness of all components (see
Fig.

$5)$.

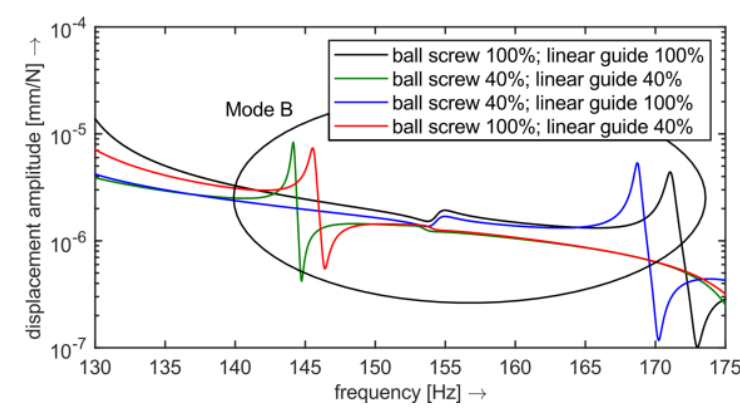

Fig. 5: Change in response to a stiffness change of all involved spring elements of the ball screw and the linear guide in the frequency range $130-175 \mathrm{~Hz}$.

The mode with the most lateral movement at the linear guides, i.e. the mode, which is most sensible to the linear guide preload, is shown in Fig. 6 .
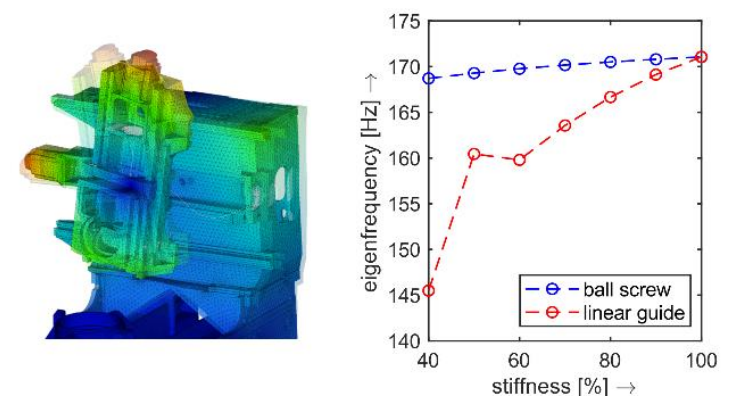

Fig. 6: Mode shape and change of natural frequency to a stiffness change of the ball screw and the linear guide for mode $B$ (see Fig. 5).

Here, it can be seen, that the natural frequency of this mode is mainly influenced by the linear guide stiffness, but not by the ball screw stiffness.

The conclusion can be drawn, that wear can be globally detected by changes in the dynamic behavior of a system. Using preliminary knowledge from an FEM analysis or even an experimental modal analysis, it is furthermore possible to not only detect wear in the form of preload loss globally, but also to refer it to specific components. Therefore, it is advisable to evaluate the characteristic modes experimentally by exciting the structure and extracting the modal parameters, which is described in the following sections.

However, evaluating the extent of the changes caused by stiffness losses leads to the interpretation, that even for significantly reduced stiffness values of the ball screw and the linear guide, the resulting changes of the natural frequency are quite small. Thus, in order to be able to monitor the condition of the machine tool, the measurement of the dynamic behavior and the extraction of the modal parameters must be very accurate, which means that changes in the natural frequency as small as $1 \mathrm{~Hz}$ must be detectable.

\section{DATA ACQUISITION: EXPERIMENTAL SETUP}

The experiments were conducted on a DMG duo Block DMC $55 \mathrm{H}$ test bench (see Fig. 7) using ball screws and linear guides with different preload levels. All investigations were carried out for the $\mathrm{x}$-axis of the machine tool. 


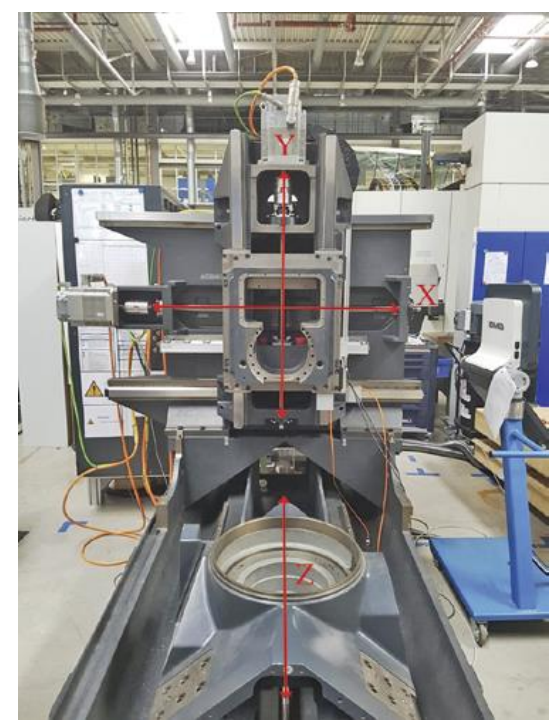

Fig. 7: Investigated test bench (machine).

The investigated components were Bosch Rexroth ball screws FEM-E-C 40x16Rx6-4 and Bosch Rexroth roller rail systems RWA 45. In order to simulate the abrasive wear in the rolling elements of both components, Bosch Rexroth AG provided ball screws and linear guides with different preload levels. The preload classes and preloads used are shown in Tab. 1.

Tab. 1: Preload classes for the ball screw and the linear guide.

\begin{tabular}{l|l} 
Ball screw class & Preload \\
\hline C3 & $2390 \mathrm{~N}$ \\
\hline C2 & $1450 \mathrm{~N}$ \\
\hline C1 & $950 \mathrm{~N}$ \\
\hline Roller rail systems class & Average Preload \\
\hline C3 & $12840 \mathrm{~N}$ \\
\hline C2 & $8965 \mathrm{~N}$ \\
\hline C1 & $4200 \mathrm{~N}$
\end{tabular}

There are three different preload classes for the ball screw and the linear guide in order to simulate three different wear states:

- $\quad$ C3 with highest preload level (new)

- $\quad$ C2 with medium preload level (medium wear)

- $\quad$ C1 with the lowest preload level (high wear)

This notation will be used in the following sections to denominate the used components in measurements. Note, that these three preload levels are commonly available for purchase showing the industrial relevance of the examined wear states. However, the classification (new, medium wear, high wear) used in this paper can only be used as a reference point since it strongly depends on the application area of the feed drive. In future work this problem is to be circumvented by comparing the simulated actual machining precision with the required precision rather than using preload classes for qualifying wear.

For the identification of the machine's modal parameters, the structure was excited by the inertial actuator SA10-V30 by CSA Engineering, which is normally used as an active vibration control system [Zaeh et al. 2017]. The actuator has a maximum force output of $90 \mathrm{~N}$. The force output needed depends on the machine's stiffness. The actuator must be strong enough to ensure a good signal to noise ratio of the vibration response. More information about the required actuator force can be found in Kleinwort et al. [2018]. The vibration response was measured by a piezoelectric acceleration sensor (Kistler 8762A10) as described in Zaeh et al. [2017]. The actuator was driven by an amplifier (BAA 120 by BEAK). A real time dSpace control system with a sampling frequency of $20 \mathrm{kHz}$ was used to control the excitation and sample the response signals. The system's response was measured over the relevant frequency range of 20 to $300 \mathrm{~Hz}$. The outputs were transferred into the frequency domain via a Fast Fourier Transformation (FFT). For all measurements in this work, the excitation point and the measurement point were located on the cross slide of the machine tool at the height of the x-axis' ball screw in order to excite all relevant modes in the considered frequency range. Actuator and sensor were kept in place via holding magnets. This position was selected according to the FEM simulation model. For the calculation of the modal parameters, the frequency response function (FRF) between the control voltage of the actuator and the measured acceleration was used.

\subsection{Test Cycle}

The measurement of the feed drive's frequency response function, which is the basis for the modal parameter extraction, is very sensitive to numerous side effects. Fig. 8 compares the results of the response of the structure for two different types of excitation signal: sinusoidal sweep and white noise. The excitation with the inertial actuator is also compared to an excitation with an impulse hammer of type 9728 A20000 by Kistler.

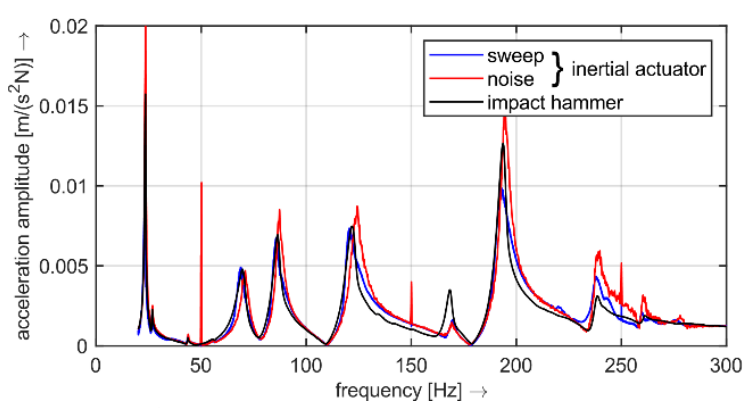

Fig. 8: Comparison of measured FRFs for differen excitation signals with the machine stationary.

It can be seen from the different responses, that the machine tool behavior is non-linear. The peaks at the multiples of $50 \mathrm{~Hz}$ indicate disturbances caused by the supply power frequency and can be neglected.

Fig. 9 shows dynamic measurement results (FRFs) conducted on the very same position for all axes and using the inertial actuator. The only change between the measurements was moving the $\mathrm{x}$-axis back and forth randomly.

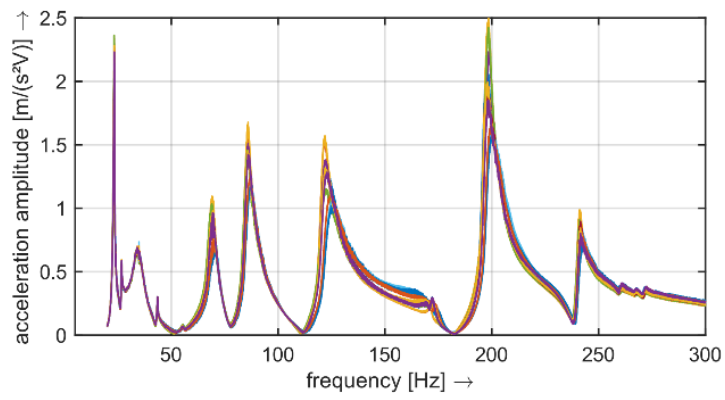

Fig. 9: Reproducibility of dynamic response measurements with respect to axes movements.

It can be seen, that there are fluctuations in all modes above $50 \mathrm{~Hz}$, and that these fluctuations are significantly larger than the changes caused by feed drive wear (see 
simulation results in section 4). In order to mitigate those effects and measure the pure machine tool dynamics, a specific test cycle was developed and implemented. For all following measurements, sinusoidal sweeps were used for two reasons: First, this allows the excitation to be fully automated requiring no manual interaction (in contrast to impact tests). Second, the signal is fully deterministic guaranteeing the highest possible reproducibility of the measurements (in contrast to random noise excitation).

Since the fluctuations are position dependent and decrease with increasing temperature, their cause is assumed to be frictional effects. In order to overcome these effects, a distinct test cycle shown schematically in Fig. 10 was designed, which was used for all following measurements. Here, a linear velocity at the linear guide is superimposed on the velocity resulting from the excitation (e.g. a sinus sweep).

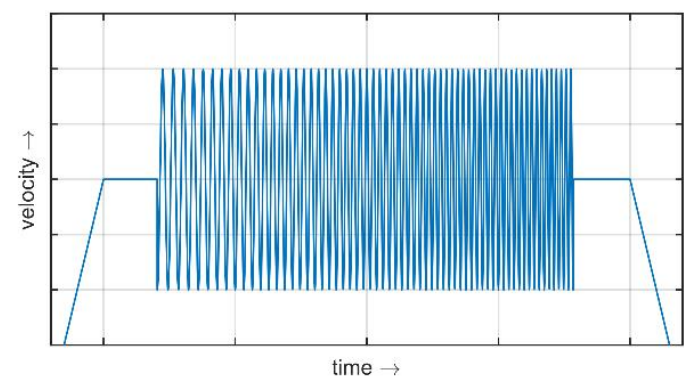

Fig. 10: Feed drive test cycle (schematically).

The superimposed linear velocity was chosen to be $100 \mathrm{~mm} / \mathrm{min}$, which is high enough to avoid the friction effects, but low enough to avoid an influence from the position dependency of the feed drive axis. Using this test cycle, a better reproducibility of the measurements can be ensured (see Fig. 11).

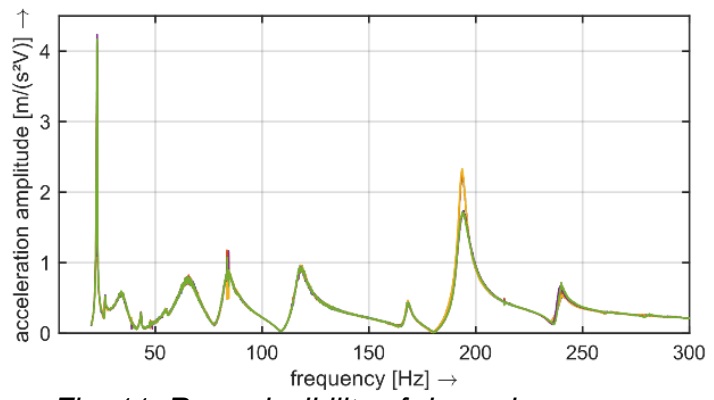

Fig. 11: Reproducibility of dynamic response measurements with superimposed linear velocity.

Going back to the comparison of different excitation signals (see Fig. 8), Fig. 12 shows, that the test cycle also reduces the deviations resulting from the different excitation sources, which proves its effectiveness.

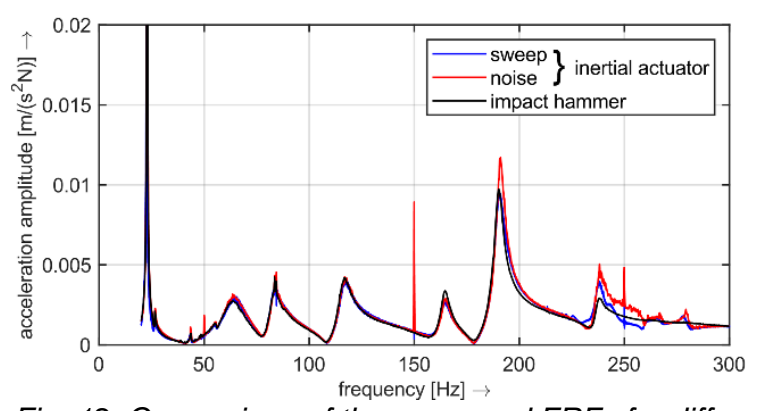

Fig. 12: Comparison of the measured FRFs for different excitation signals with superimposed linear velocity.

\subsection{Analyses of the Response Function}

By comparing the measured system's dynamic behavior (FRF) for different ball screw preloads, but fixed linear guide preloads (Fig. 14(a)) and for different linear guide preloads, but fixed ball screw preload (Fig. 14(b)), respectively, it is clear, that the three modes at $66.5 \mathrm{~Hz}, 83 \mathrm{~Hz}$ and $117 \mathrm{~Hz}$ are sensitive to ball screw wear and the two modes at $168 \mathrm{~Hz}$ and $193 \mathrm{~Hz}$ are sensitive to linear guide wear.

The variation of the ball screw preload class shows that the stiffness of the ball screw indeed has an influence on the dynamic behavior of the machine tool. However, the difference between the preload classes C3 and C2 is so small for most modes that it is difficult to detect it. The preload class $\mathrm{C} 1$, on the other hand, has significantly lower natural frequencies and higher amplitude values at the significant modes. This is in line with the observations described in section 4, where simulations showed that only high preload losses at the ball screw lead to detectable changes in the modal parameters.

Similarly, the influence of the stiffness of the linear guide can be analyzed as well. The ball screw preload state was kept constant at $\mathrm{C} 1$. Again, the simulation results from section 4 can be confirmed, since the measured FRFs showed much more sensitivity to the linear guide stiffness than to the ball screw stiffness.

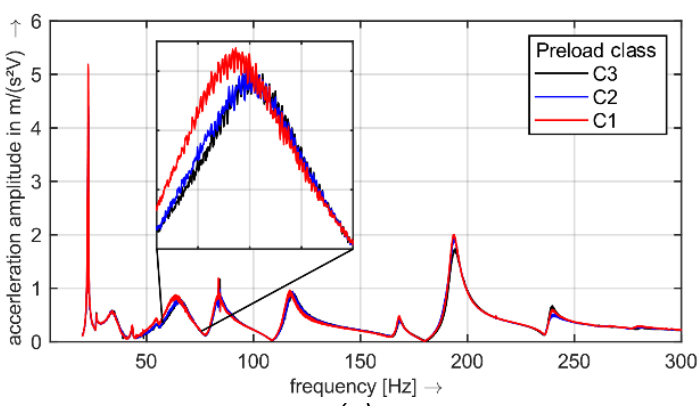

(a)

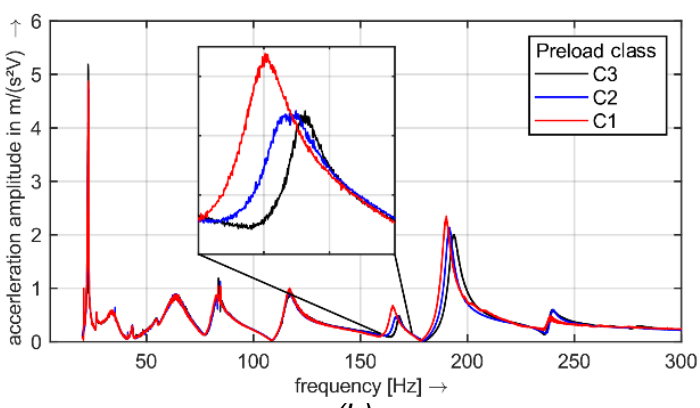

(b)

Fig. 13: Changes of the dynamic behavior for varying ball screw preload (a) and varying linear guide preload (b).

\section{FEATURE EXTRACTION: MODAL PARAMETERS}

Knowing the importance and the benefit of the modal parameters theoretically described in section 3, two methods were implemented for extracting features from the measurements of the dynamic behavior (FRF) of the feed drive using the test cycle presented in section 5: First, a combination of the $3 \mathrm{~dB}$-method and peak-picking was used for estimating the modal parameters [Ewins 2000]. Here, it is assumed that the individual peaks of the transfer function are well separated leading to a decrease in amplitude of at least $1 / \sqrt{2}$ for each peak. Two features are to be derived here: First, the natural frequency is estimated to be the frequency with the highest amplitude $\left(\hat{f}_{d, i}\right)$. Second, the 
peak amplitude itself serves as another feature $\left(H\left|\omega_{d, i}\right|\right)$. Fig. 14 shows the resulting frequencies and amplitudes for the eight most significant modes measured.

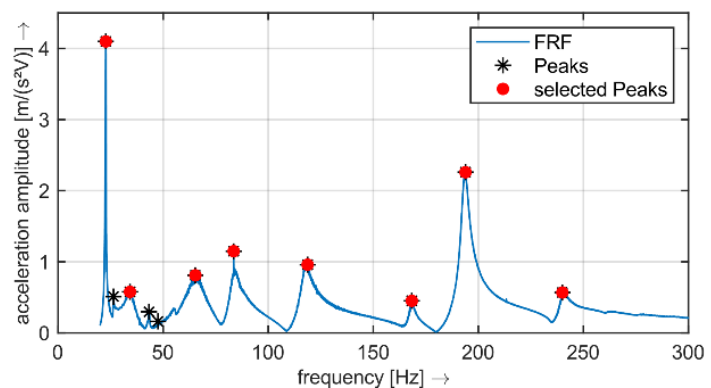

Fig. 14: Identified modes using the 3dB-method.

The second method implemented here is the polyreference least-squares complex frequency-domain method (PolyMAX). It is a global MDOF method meaning that the modes in this case do not necessarily need to be well separated. Thus, it can also be applied when the peak-picking approach fails due to interfering modes, making it more robust for application in real production environments. With this method, an accurate estimate of the modal parameters can be found. The poles of the FRF described in equation (3) were calculated with the polyreference least-squares complex frequency method [Guillaume et al. 2003]. To determine the modal coefficients, the least-squares frequency domain algorithm was used.

With the PolyMAX-algorithm a large number of possible FRF poles was found. The number of found poles increases with increasing predefined maximum model order (here 25). All unstable poles, i.e. poles leading to negative eigenfrequencies or damping, were immediately discarded. The remaining poles were then grouped by the eigenmodes they belong to. Each group is approximated by a vertical line in Fig. 15, i.e. there are eight relevant groups. As a last step, for each group the pole with the highest value of the expected frequency response assurance criterion (FRAC) was selected. Fig. 15 shows the identified modes and the resulting, well-fitted model of the measured transfer function.

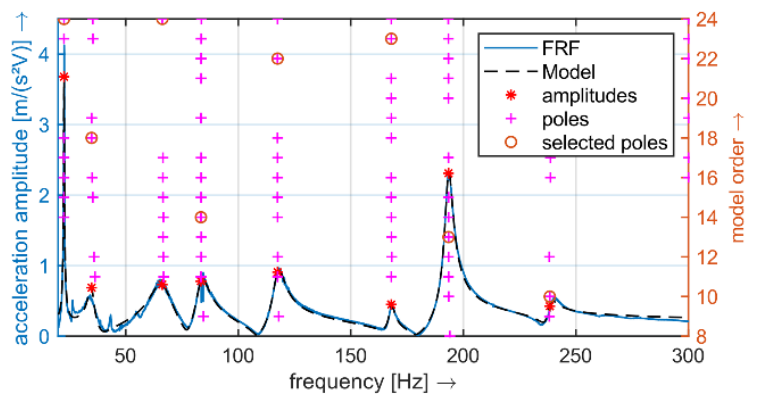

Fig. 15: Identified modes and fit function using the PolyMAX algorithm.

\section{ANAYLSIS OF VARIANCE}

The analysis of variance (ANOVA) is a method for determining the dependency of response variables to a set of input variables [Dean et al. 2017]. The goal is to distinguish real effects from apparent effects caused by measurement noise. The input variables within this work are the preload classes of the ball screw and the linear guide (see Tab. 1). The output variables are the modal features derived from the measured FRFs in section 6 . Thus, for each feature (or quality feature $\gamma$ in terms of the
ANOVA) a model equation for analysis of variance with two (input) factors each is considered here:

$$
\gamma_{i j}=\mu+\alpha_{i}+\beta_{j}+(\alpha \beta)_{i j}+\varepsilon_{i j}
$$

Here, $\mu$ is the mean value of all measurements, $\alpha$ the effect of the preload class of the ball screw, $\beta$ the effect of the preload class of the linear guide, $\alpha \beta$ the influence of the interaction and $\varepsilon$ an error not covered by the model. The indices $i$ and $j$ describe the preload class level (see Tab. 1). Altogether, 24 model equations were set up for the PolyMAX method (natural frequency, modal damping and amplitude at the natural frequency for eight modes) and 16 for the peak-picking method (peak amplitude and frequency).

An input factor is considered to be significant if the change is not compatible with the null hypothesis of a purely random deviation, i.e. there is no effect of the input factor [Dean et al. 2017]. The p-value describes the probability of seeing the measured effect of the input parameter under the assumption of a valid null hypothesis. This also corresponds to the risk of unfairly rejecting the null hypothesis. Thus, a sufficiently small $p$-value suggests that a factor has a significant effect on the considered outcome.

In order to validate the approach presented here, the factorial screening depicted in Tab. 2 was conducted. Each " $x$ " denotes a tested combination. It can be seen, that the experiment design represents a fractional factorial screening design with one repetition and a full factorial screening design with only partial repetition, respectively. The goal was to find qualitative relationships between preload loss of the ball screw and the linear guide and the system's modal parameters.

Tab. 2: Design of experiments.

\begin{tabular}{|c|c|c|c|c|}
\cline { 3 - 5 } \multicolumn{2}{c|}{} & \multicolumn{3}{|c|}{ Linear guide preload } \\
\cline { 3 - 5 } \multicolumn{2}{c|}{} & $\mathrm{C} 1$ & $\mathrm{C} 2$ & $\mathrm{C} 3$ \\
\hline \multirow{2}{*}{$\begin{array}{c}\text { Ball } \\
\text { screw } \\
\text { preload }\end{array}$} & $\mathrm{C} 1$ & $\mathrm{Xx}$ & $\mathrm{x}$ & $\mathrm{xx}$ \\
\cline { 2 - 5 } & $\mathrm{C} 2$ & $\mathrm{x}$ & $\mathrm{xx}$ & $\mathrm{xx}$ \\
\cline { 2 - 5 } & $\mathrm{C} 3$ & $\mathrm{xx}$ & $\mathrm{x}$ & $\mathrm{xx}$ \\
\hline
\end{tabular}

By calculating modal parameters for each configuration within Tab. 2, the causal connection with the preload condition for the eight most significant modes was evaluated. Tab. 3 shows the calculated $p$-values for the PolyMAX method $\left(f_{d, i}, d_{i}, H\left|\omega_{d, i}\right|\right)$. The influence is regarded as significant, if the calculated $p$-value is smaller than $3 \%$. Those modal parameters are highlighted by printing them bold.

Tab. 3: $p$-values for PolyMAX method.

\begin{tabular}{|c|c|c|c|}
\hline & $\alpha_{i}$ & $\beta_{j}$ & $(\alpha \beta)_{i j}$ \\
\hline$f_{d, 1}$ & 0.14588 & 0.95842 & 0.81597 \\
\hline$f_{d, 2}$ & 0.35223 & 0.53488 & 0.52991 \\
\hline$f_{d, 3}$ & 0.00008 & 0.03849 & 0.73639 \\
\hline$f_{d, 4}$ & 0.00001 & 0.03590 & 0.20068 \\
\hline$f_{d, 5}$ & 0.00017 & 0.13153 & 0.60689 \\
\hline$f_{d, 6}$ & 0.42266 & 0.00009 & 0.60933 \\
\hline$f_{d, 7}$ & 0.78108 & 0.00002 & 0.56255 \\
\hline$f_{d, 8}$ & 0.49767 & 0.11745 & 0.83995 \\
\hline$d_{1}$ & 0.00789 & 0.28051 & 0.97647 \\
\hline$d_{2}$ & 0.90601 & 0.95080 & 0.63993 \\
\hline$d_{3}$ & 0.06550 & 0.73954 & 0.79394 \\
\hline
\end{tabular}




\begin{tabular}{c|lll}
$d_{4}$ & $\mathbf{0 . 0 1 6 6 6}$ & 0.04275 & 0.03085 \\
$d_{5}$ & $\mathbf{0 . 0 0 1 2 0}$ & 0.04902 & 0.54342 \\
$d_{6}$ & 0.30187 & $\mathbf{0 . 0 2 6 5 8}$ & 0.86111 \\
$d_{7}$ & 0.78151 & 0.81671 & 0.54243 \\
$d_{8}$ & 0.62993 & 0.57533 & 0.58134 \\
$H\left|\omega_{d, 1}\right|$ & $\mathbf{0 . 0 0 1 1 6}$ & 0.04671 & 0.81931 \\
$H\left|\omega_{d, 2}\right|$ & 0.64055 & 0.99837 & 0.88207 \\
$H\left|\omega_{d, 3}\right|$ & $\mathbf{0 . 0 0 0 0 6}$ & $\mathbf{0 . 0 0 4 8 1}$ & 0.32469 \\
$H\left|\omega_{d, 4}\right|$ & $\mathbf{0 . 0 2 9 3 1}$ & 0.29018 & 0.16988 \\
$H\left|\omega_{d, 5}\right|$ & 0.41152 & 0.48103 & 0.69923 \\
$H\left|\omega_{d, 6}\right|$ & 0.42704 & $\mathbf{0 . 0 0 2 4 6}$ & 0.31004 \\
$H\left|\omega_{d, 7}\right|$ & 0.41911 & 0.39495 & 0.59177 \\
$H\left|\omega_{d, 8}\right|$ & 0.20555 & 0.61734 & 0.67188
\end{tabular}

It can be seen, that the effect of the preload class of the ball screw $\alpha$ is high for the modes $1,3,4$ and 5 whereas the effect of the preload class of the linear guide $\beta$ is significant for the modes 3, 6 and 7 even though not always every modal parameter of each mode has a smaller value than $3 \%$. The interaction $\alpha \beta$ shows no influence. Damping and amplitude of mode 7 is not statistically significant, because even though the test cycle is used (see section 5.1) there are still fluctuations at this mode (see Fig. 11).

By using the 3dB-method, the following $p$-values for the estimated modal parameters $\left(\hat{f}_{d, i}, H\left|\omega_{d, i}\right|\right)$ were calculated (Tab. 4):

Tab. 4: p-values for the 3dB-method.

\begin{tabular}{c|ccc} 
& $\alpha_{i}$ & $\beta_{j}$ & $(\alpha \beta)_{i j}$ \\
$\hat{f}_{d, 1}$ & 0.188667 & 0.632457 & 0.934276 \\
$\hat{f}_{d, 2}$ & 0.269441 & 0.037022 & 0.545050 \\
$\hat{f}_{d, 3}$ & $\mathbf{0 . 0 0 4 2 8 1}$ & 0.529506 & 0.935150 \\
$\hat{f}_{d, 4}$ & 0.229306 & 0.190635 & 0.390704 \\
$\hat{f}_{d, 5}$ & $\mathbf{0 . 0 0 0 9 1 2}$ & $\mathbf{0 . 0 2 8 6 9 2}$ & 0.667283 \\
$\hat{f}_{d, 6}$ & 0.430676 & $\mathbf{0 . 0 0 0 0 0 3}$ & 0.238926 \\
$\hat{f}_{d, 7}$ & 0.154078 & $\mathbf{0 . 0 0 0 0 5 9}$ & 0.114517 \\
$\hat{f}_{d, 8}$ & 0.925905 & 0.067478 & 0.346550 \\
$H\left|\omega_{d, 1}\right|$ & $\mathbf{0 . 0 0 0 2 3 4}$ & $\mathbf{0 . 0 0 7 9 4 8}$ & 0.857778 \\
$H\left|\omega_{d, 2}\right|$ & 0.990945 & 0.431978 & 0.783258 \\
$H\left|\omega_{d, 3}\right|$ & $\mathbf{0 . 0 0 0 0 7 1}$ & $\mathbf{0 . 0 0 5 9 8 4}$ & 0.282532 \\
$H\left|\omega_{d, 4}\right|$ & 0.348038 & 0.655077 & 0.773524 \\
$H\left|\omega_{d, 5}\right|$ & 0.329480 & 0.634335 & 0.579398 \\
$H\left|\omega_{d, 6}\right|$ & 0.443097 & $\mathbf{0 . 0 0 2 1 2 1}$ & 0.193793 \\
$H\left|\omega_{d, 7}\right|$ & 0.473215 & 0.543229 & 0.656371 \\
$H\left|\omega_{d, 8}\right|$ & 0.086454 & $\mathbf{0 . 0 1 1 2 1 7}$ & 0.073479
\end{tabular}

Mode 4 is no longer significant for ball screw preload because of errors in identifying the peak at $83 \mathrm{~Hz}$ (see Fig. 14).

For the feed drive system with well-separated modes as considered here (see section 5), the conducted ANOVA leads to the conclusion that both the peak-picking and the PolyMAX approach show roughly the same dependencies of the ball screw's and linear guide's preload on the modal parameters. However, it is expected that the PolyMAX approach will outperform peak-picking when the investigated system has several interfering modes.

\section{SUMMARY AND OUTLOOK}

In this paper, a method was presented for monitoring the condition of feed drives by looking at the dynamic behavior. It was shown, how results from a simulated sensitivity analysis can be used not only to detect, but also to locate feed drive wear. Furthermore, requirements on the precision of the used methods could be derived leading to the development of a distinct test cycle for overcoming non-linear effects and for achieving reproducible measurements of the feed drive's dynamic behavior. Two methods were presented for deriving wear sensitive features based on the modal parameters. Lastly, a variance analysis was presented, showing the significance of the derived features, and confirming the simulation results, i.e. that the dynamic behavior cannot only detect wear, but can also locate it. However, in this work only the influence of the preload on the modal parameters was shown - not how to estimate the current preload state from the modal parameters. Thus, further work needs to be done to develop models able to predict the current condition of feed drive components from dynamic measurements.

\section{ACKNOWLEDGMENTS}

This work was supported by the European Commission within the project "PreCoM - Predictive Cognitive Maintenance Decision Support System" under Grant Agreement No. 768575.

\section{REFERENCES}

[Altintas 2012] Altintas, Y.: Manufacturing automation. Metal cutting mechanics, machine tool vibrations, and CNC design. $2^{\text {nd }}$ ed. : Cambridge, New York: Cambridge University Press 2012. ISBN: 0521172470.

[Chang et al. 2010] Chang, J.-L.; Chao, J.-A.; Huang, Y.C.; Chen, J.-S.: Prognostic experiment for ball screw preload loss of machine tool through the Hilbert-Huang Transform and Multiscale entropy method. (Hrsg.): The 2010 IEEE International Conference on Information and Automation, 2010 International Conference on Information and Automation (ICIA). Harbin, China, 20.06.2010 23.06.2010: IEEE 2010, pp. 376-380. ISBN: 978-1-42445701-4.

[Dean et al. 2017] Dean, A.; Voss, D.; Draguljić, D.: Design and Analysis of Experiments. Cham: Springer International Publishing 2017. ISBN: 978-3-319-52248-7.

[Ewins 2000] Ewins, D. J.: Modal testing. Theory, practice and application. 2. ed. : Baldock: Research Studies Press 2000. ISBN: 9780863802188

[Farrar et al. 2012] Farrar, C. R.; Worden, K.: Structural Health Monitoring. Chichester, UK: John Wiley \& Sons, Ltd 2012. ISBN: 9781118443118.

[Feng et al. 2012] Feng, G.-H.; Pan, Y.-L.: Investigation of ball screw preload variation based on dynamic modeling of a preload adjustable feed-drive system and spectrum analysis of ball-nuts sensed vibration signals. International Journal of Machine Tools and Manufacture 52 (2012) 1, pp. 85-96.

[Guillaume et al. 2003] Guillaume, P.; Verboven, P.; Cauberghe, B.; Vanlanduit, S.; Parloo, E.; Sitter, G. de: Frequency-Domain System Identification Techniques for 
Experimental and Operational Modal Analysis. IFAC Proceedings Volumes 36 (2003) 16, pp. 1609-1614.

[Hillenbrand et al. 2018] Hillenbrand, J.; Spohrer, A.; Fleischer, J.: Zustandsüberwachung bei Kugel gewindetrieben. Integration von DMS-Sensorik in Kugelgewindetriebemuttern. wt Werkstattstechnik online 108 (2018) 7/8, pp. 493-498.

[Kleinwort et al. 2018] Kleinwort, R.; Weishaupt, P.; Zaeh, M. F.: Simulation-Based Dimensioning of the Required Actuator Force for Active Vibration Control. International Journal of Automation Technology 12 (2018) 5 , pp. 658-668.

[Maier 2015] Maier, D.: Sensorlose online Zustandserfassung von Vorschubantriebskomponenten in Werkzeugmaschinen. (Zugl.: Stuttgart, Univ., Diss., 2014)Stuttgart: IMA 2015. ISBN: 9783936100587.

[Semm et al. 2019] Semm, T.; Nierlich, M. B.; Zaeh, M. F.: Substructure coupling of a machine tool in arbitrary axis positions considering local linear damping models. Journal of Manufacturing Science and Engineering (2019).

[Semm et al. 2018] Semm, T.; Spannagl, M. F.; Zaeh, M. F.: Dynamic substructuring of machine tools considering local damping models. Procedia CIRP 77 (2018), pp. $670-674$.

[Tsai et al. 2014] Tsai, P. C.; Cheng, C. C.; Hwang, Y. C.: Ball screw preload loss detection using ball pass frequency. Mechanical Systems and Signal Processing 48 (2014) 1-2, pp. 77-91.

[Verl et al. 2009] Verl, A.; Heisel, U.; Walther, M.; Maier, D.: Sensorless automated condition monitoring for the control of the predictive maintenance of machine tools. CIRP Annals 58 (2009) 1, pp. 375-378.

[Walther 2011] Walther, M.: Antriebsbasierte

Zustandsdiagnose von Vorschubantrieben. (Zugl.:

Stuttgart, Univ., Diss., 2011)Heimsheim: Jost-Jetter 2011. ISBN: 9783939890775.

[Zaeh et al. 2017] Zaeh, M. F.; Kleinwort, R.; Fagerer, P.; Altintas, Y.: Automatic tuning of active vibration control systems using inertial actuators. CIRP Annals 66 (2017) 1, pp. 365-368.

[Zaeh et al. 2004] Zaeh, M. F.; Oertli, T.; Milberg, J.: Finite Element Modelling of Ball Screw Feed Drive Systems. CIRP Annals 53 (2004) 1, pp. 289-292.

[Zaeh et al. 2019] Zaeh, M. F.; Rebelein, C.; Semm, T.: Predictive simulation of damping effects in machine tools. CIRP Annals 68 (2019).

[Zhai et al. 2018] Zhai, S.; Reinhart, G.: Predictive Maintenance als Wegbereiter für die instandhaltungsgerechte Produktionssteuerung. ZWF Zeitschrift für wirtschaftlichen Fabrikbetrieb 113 (2018) 5, pp. 298-301. 